\title{
Clinical features and treatment strategies for older prostate cancer patients with bone metastasis
}

\author{
Cheng Yang ${ }^{1}$, Gui-Sheng $\mathrm{Qi}^{1}$, Rui-Ming Rong ${ }^{1}$ and Jian $\mathrm{He}^{2}$
}

To identify the clinical features and independent predictors of survival in older patients with bone metastasis from prostate cancer (PCa). We retrospectively analysed 205 older patients with bone metastases from PCa between 1997 and 2012 . The Kaplan-Meier method was used with the log-rank test for survival rate calculations and to evaluate each variable. Multivariate analysis was performed with the Cox regression model. The chi-squared test was used to compare survival rates between older and younger $(n=197)$ patients. All patients were followed up. The 1-, 2-, 3- and 5-year survival rates were $95.5 \%, 77.5 \%, 68.5 \%$ and $33.7 \%$, respectively. Gleason score, radiotherapy of the primary tumour, the number of bone metastases, the alkaline phosphatase alkaline phosphatase (ALP) level, organ metastasis and regional lymph node metastasis were associated with the survival rates. Multivariate Cox regression analysis showed that Gleason score at diagnosis of the primary tumour was a significant predictor of overall survival following the diagnosis of bone metastases. In addition, the overall survival rates of older patients were higher compared with younger patients, but older patients who underwent radiotherapy had higher mortality. These data may serve as a guide for creating clinical prediction models in further studies.

Asian Journal of Andrology (2013) 15, 759-763; doi:10.1038/aja.2013.111; published online 16 September 2013

Keywords: bone metastasis; older; prognostic analysis; prostate cancer (PCa); clinical feature

\section{INTRODUCTION}

Prostate cancer ( $\mathrm{PCa})$ is the most commonly diagnosed malignancy among males in developed countries and the second leading cause of cancer-related mortality. ${ }^{1}$ Currently, the incidence is rapidly increasing in developing Asian counties such as China. It is the only solid tumour diagnosed with its first recurrence as bone metastases instead of visceral metastases. PCa frequently metastasizes to bone, and treatment with androgen deprivation leads to accelerated bone loss, resulting in clinically relevant skeletal complications associated with disabling symptoms. ${ }^{2}$ Among patients who die from PCa, the incidence of skeletal involvement appears to be $>85 \%$. ${ }^{3,4}$

Eisenberger and Hussain et al. ${ }^{5}$ identified baseline covariates that predict the long-term survival of patients with metastatic PCa based on the Southwest Oncology Group criteria. Minimal disease, better Southwest Oncology Group performance status, no bone pain, lower Gleason score and lower prostate-specific antigen (PSA) level were independent factors associated with longer survival. ${ }^{5}$ In our previous study, we found that bone metastasis of PCa was more common in older males, ${ }^{6}$ but no prognostic factors for these PCa patients were identified. In this retrospective study, the sample size was enlarged and 205 PCa patients older than 70 with bone metastases were included. The patients' clinical features and main prognostic factors, including age, Gleason score, stage, endocrine therapy, castration, radiotherapy, number of bone lesions, PSA and alkaline phosphatase (ALP) levels, neighbouring organ metastasis, regional and remote lymph node metastases, invasion of the surrounding parenchyma and other visceral metastases, were analysed to determine treatment strategies and predict the reactions of the older patients to treatment.

\section{MATERIALS AND METHODS}

Patient collection

A total of 205 older and 197 younger patients with bone metastases from PCa who were treated between 1997 and 2012 at Zhongshan Hospital, Fudan University, were included (Table 1). The data for the young patients were partially derived from our previous study. ${ }^{6}$ All of the patients were confirmed as having primary PCa by pathological diagnosis and were diagnosed with bone metastasis through radioisotope scanning or magnetic resonance imaging.

Collection of clinical and pathological data

Patient age at diagnosis, Gleason score and clinical stage at initial diagnosis, treatment for primary prostate lesions, including endocrine therapy, radiotherapy and chemotherapy, number of bone lesions, PSA and ALP levels, organ metastasis sites, regional and remote lymph node metastases, follow-up duration and survival status were retrospectively collected and reviewed. Serum PSA values were determined in the same laboratory using the ELISA method. The normal range was $0-4 \mathrm{ng} \mathrm{ml}^{-1}$. The PSA values of the patients were characterized as 0 $4 \mathrm{ng} \mathrm{ml}^{-1}, 4-20 \mathrm{ng} \mathrm{ml}^{-1}$ and greater than $20 \mathrm{ng} \mathrm{ml}^{-1}$. In the pathological examinations of the patients, Gleason scores were characterized as $2-4,5-7$ and $8-10$. When determining the number of metastases by radioisotope scanning, the number of metastases in each vertebra and 
Table 1 Baseline characteristics of older and younger patients

\begin{tabular}{|c|c|c|c|}
\hline & Older ${ }^{a}$ & Younger $^{a}$ & $\mathrm{P}$ \\
\hline Age (year) & $75.04 \pm 4.60$ & $63.32 \pm 4.77$ & $<0.001$ \\
\hline PSA when bone metastasis was observed $\left(\mathrm{ng} \mathrm{ml}^{-1}\right)$ & $75.70 \pm 7.14$ & $220.32 \pm 51.16$ & 0.009 \\
\hline Gleason score when diagnosed as having PCa & $7.77 \pm 1.22$ & $7.92 \pm 1.27$ & 0.589 \\
\hline
\end{tabular}

Abbreviations: ALP, alkaline phosphatase; PSA, prostate-specific antigen.

${ }^{a}$ Data are presented as the mean \pm s.e.m.

rib metastasis was considered as one metasitasis; in the statistical evaluation, the number of bone metastases was assessed as either single or multiple. The study design was approved by the institutional ethics review board of Zhongshan Hospital, Fudan University.

\section{Follow-up and statistical methods}

The survival time was calculated from the date of the first diagnosis of bone metastasis to the date of death or last follow-up. Univariate and multivariate analyses were performed using SPSS 18.0 software (IBM, Armonk, NY, USA). The Kaplan-Meier method with a log-rank test was used for survival rate calculations and to evaluate each variable. Multivariate analysis was performed with the Cox regression model, and all of the variables were entered in a single step, using backward stepwise regression (likelihood ratio). The chi-squared test was used to compare survival rates between older and younger patients. All of the tests were two sided, and $P<0.05$ was considered statistically significant.

\section{RESULTS}

\section{Patient characteristics}

For the older patients, the median age was 75 (range: 70-91) years. In this group, metastases had spread to the spine $(43.3 \%)$, and the thoracic vertebrate $(17.2 \%)$ and lumbar vertebrate $(16.8 \%)$ were the most frequently involved sites. Figure 1 shows the sites of bone metastasis observed in the older patients.

\section{Survival time analysis}

All of the patients (100\%) were followed up until the date of death or 1 August 2012. The median follow-up was 55.6 (interquartile range: 8.0175.0) months. Following the diagnosis of bone metastasis, the 1-, 2-, 3 - and 5-year survival rates were $95.5 \%, 77.5 \%, 68.5 \%$ and $33.7 \%$, respectively, with a median survival time of 55.6 (range: $8.0-175.0$ ) months. Following the diagnosis of $\mathrm{PCa}$, the 1-, 2-, 3- and 5-year

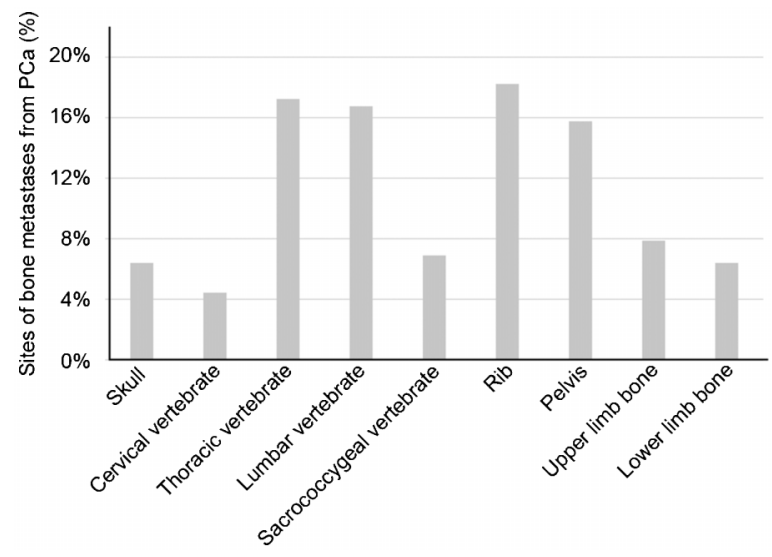

Figure 1 Sites of bone metastasis from PCa. PCa, prostate cancer. survival rates were $95.5 \%, 77.5 \%, 68.5 \%$ and $33.7 \%$, respectively, with a median survival time of 56.2 (range: 7-175) months.

\section{Prognostic factors in univariate and multivariate analyses}

The results of the univariate analysis indicated that survival was associated with Gleason score, radiotherapy of the primary tumour, the number of bone metastases, the ALP level, sites of organ metastasis and regional lymph node metastasis $(P<0.05)$. The differences for other factors were not significant $(P>0.05$, Table 2$)$.

Multivariate Cox regression analysis showed that age and Gleason score at the time of primary tumour diagnosis were significant predictors of overall survival following the diagnosis of bone metastasis (Table 3).

Comparisons of survival rates between older and younger patients The survival rates between older and younger patients were compared (figure 2). The survival rate of the older patients was significantly higher compared with the younger patients (Figure 2a). Older patients with more than $150 \mathrm{IU} \mathrm{l}^{-1}$ ALP, multiple sites of bone metastasis or neighbouring organ metastasis had a higher survival rate (Figure 2d, 2h and $2 \mathbf{k}$ ). In addition, older patients without radiotherapy of the primary tumour and regional lymph node metastasis also had a significantly higher survival rate compared with younger patients (Figure $\mathbf{2 f}$ and $2 \mathbf{j}$ ). Similarly, older patients with single-bone metastasis had a higher survival rate (Figure $2 \mathbf{g}$ ). Interestingly, the older patients who received radiotherapy of the primary tumour demonstrated a lower survival rate than the younger patients (Figure 2e). Other factors did not significantly influence the survival rate between the two groups (Figure $2 \mathbf{b}, 2 \mathrm{c}, 2 \mathbf{i}$ and $2 \mathbf{l}$ ).

\section{DISCUSSION}

Although the early diagnostic methods for PCa are advanced (e.g., PSA screening, high-resolution magnetic resonance imaging, ultrasonography), approximately $10 \%-20 \%$ of PCa patients are initially diagnosed because of bone metastasis. ${ }^{7}$ The majority of patients with localized early stage disease do not develop metastasis and do not die of prostate cancer. However, among patients with castration-resistant metastatic prostate cancer, more than $80 \%$ of patients present bone metastases, which is the most common site of metastasis in this group. ${ }^{2}$

The patients in our study ranged in age from 70 to 91 years, and the data demonstrated that most bone metastases of PCa did not extend into the adjacent organs. The clinical use of PSA to accurately predict prognosis is recognized as inadequate because PSA is prostate specific but not PCa specific, as it is known to be increased by other prostate diseases such as benign hyperplasia as well as by inflammation, transrectal ultrasound examination, biopsy and transurethral prostatectomy. However, due to a lack of other more specific markers, serum PSA is still suggested for PCa diagnosis and follow-up. Although Glass et al. ${ }^{8}$ stated that PSA was an independent predictor of metastatic $\mathrm{PCa}$, other researchers have reported that the pre-treatment PSA level does not have a statistically significant effect on survival in patients with 
Table 2 Univariate analysis for survival

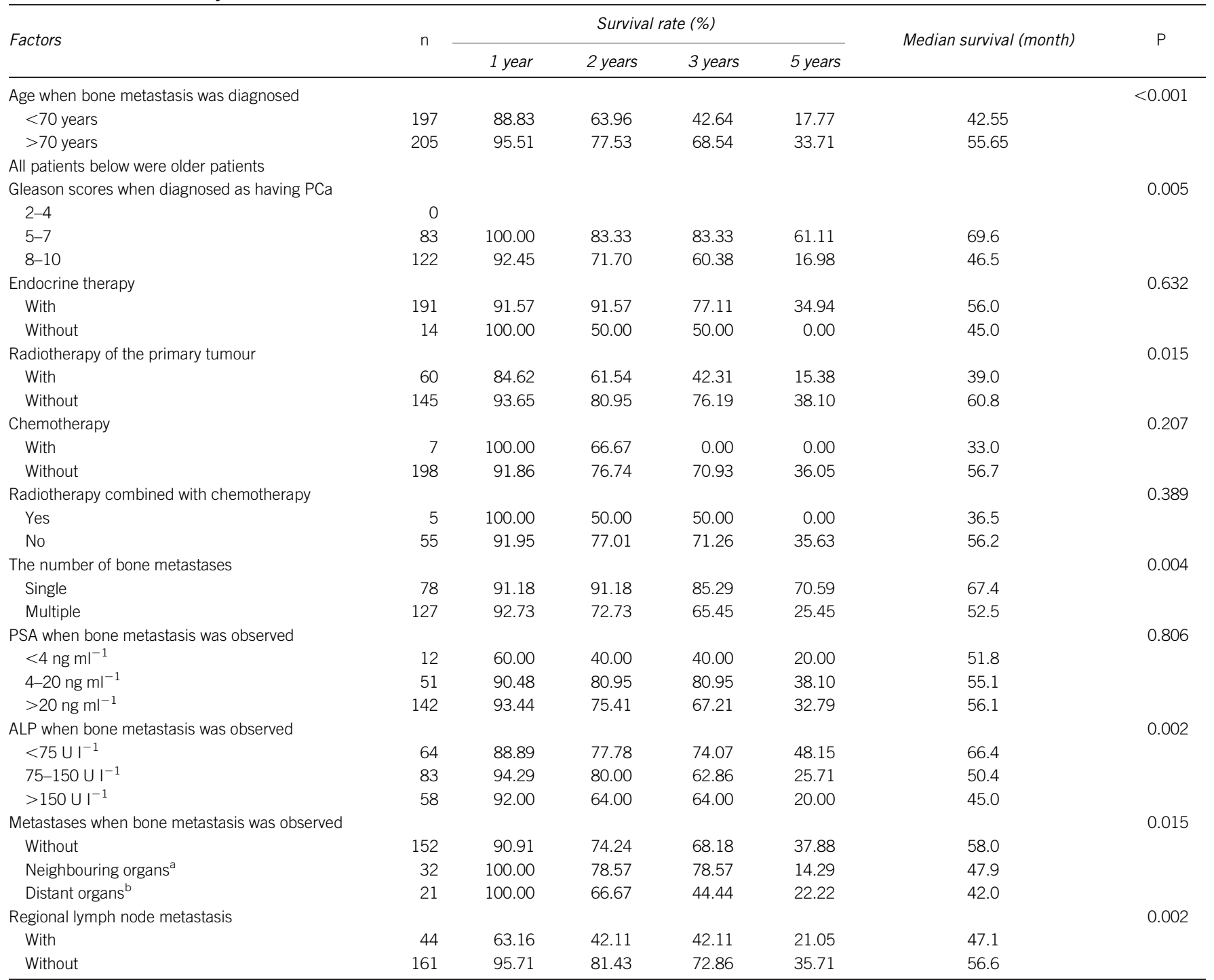

Abbreviations: ALP, alkaline phosphatase; PSA, prostate-specific antigen.

${ }^{a}$ Defined as an organ around the prostate, such as the bladder, spermatophores, urethra and rectum.

${ }^{\mathrm{b}}$ Defined as brain, lung and liver metastases.

bone metastases. ${ }^{9,10}$ A study evaluating a series of Southwest Oncology Group phase III trials revealed that overall survival was significantly improved in the post-PSA era. ${ }^{11}$. To increase the sensitivity and specificity of PSA, some new indexes were developed, including PSA density, velocity, doubling time and the free to total PSA ratio, which have increased the significance of serum PSA in diagnosing PCa. In the current study, the PSA level at the time bone metastases were observed was identified as a significant survival predictor.

Table 3 Multivariate analysis for survival

\begin{tabular}{lcccc}
\hline Factors & $\beta$ & s.e. & $R R$ & $P$ \\
\hline Age & -1.387 & 0.751 & 0.250 & 0.045 \\
Gleason scores when diagnosed as PCa & -1.139 & 0.611 & 0.249 & 0.023 \\
ALP when bone metastasis was observed & -1.151 & 0.740 & 0.316 & 0.120 \\
Regional lymph node metastasis & -0.091 & 0.063 & 0.921 & 0.190 \\
\hline
\end{tabular}

Abbreviations: ALP, alkaline phosphatase; $\beta$, coefficient of regression; $\mathrm{PCa}$, prostate cancer; RR, relative risk; s.e., standard error.
In accordance with our results, the serum ALP level has been found to be an important prognostic factor in many studies. ${ }^{9,12}$ In the current study, the median overall survival of patients with Gleason scores $>8$ was significantly shorter than that of the lower scoring patients, which is consistent with our previous study. ${ }^{6}$ Cheville et al. ${ }^{13}$ and Yigitbasi et al. ${ }^{9}$ have also reported that among patients with bone metastases, the Gleason score of the primary prostate adenocarcinoma is strongly associated with patient outcome. Saeter et al. ${ }^{14}$ found that the survival of patients with soft tissue or non-regional lymph node metastasis was similar to that of patients presenting with skeletal metastasis only, but few studies have discussed the prognostic value of these factors. We have previously demonstrated that regional lymph node metastasis is an independent predictor in metastatic PCa patients; however, in the present study, it was not predictive for older patients with metastatic PCa.

To prolong survival and improve quality of life, comprehensive therapy is necessary. Radiotherapy is a very effective method for treating bone metastases. Interestingly, our previous study demonstrated 


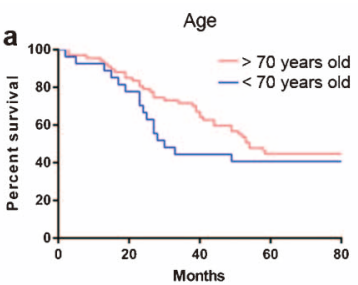

ALP when bone metastases were observed
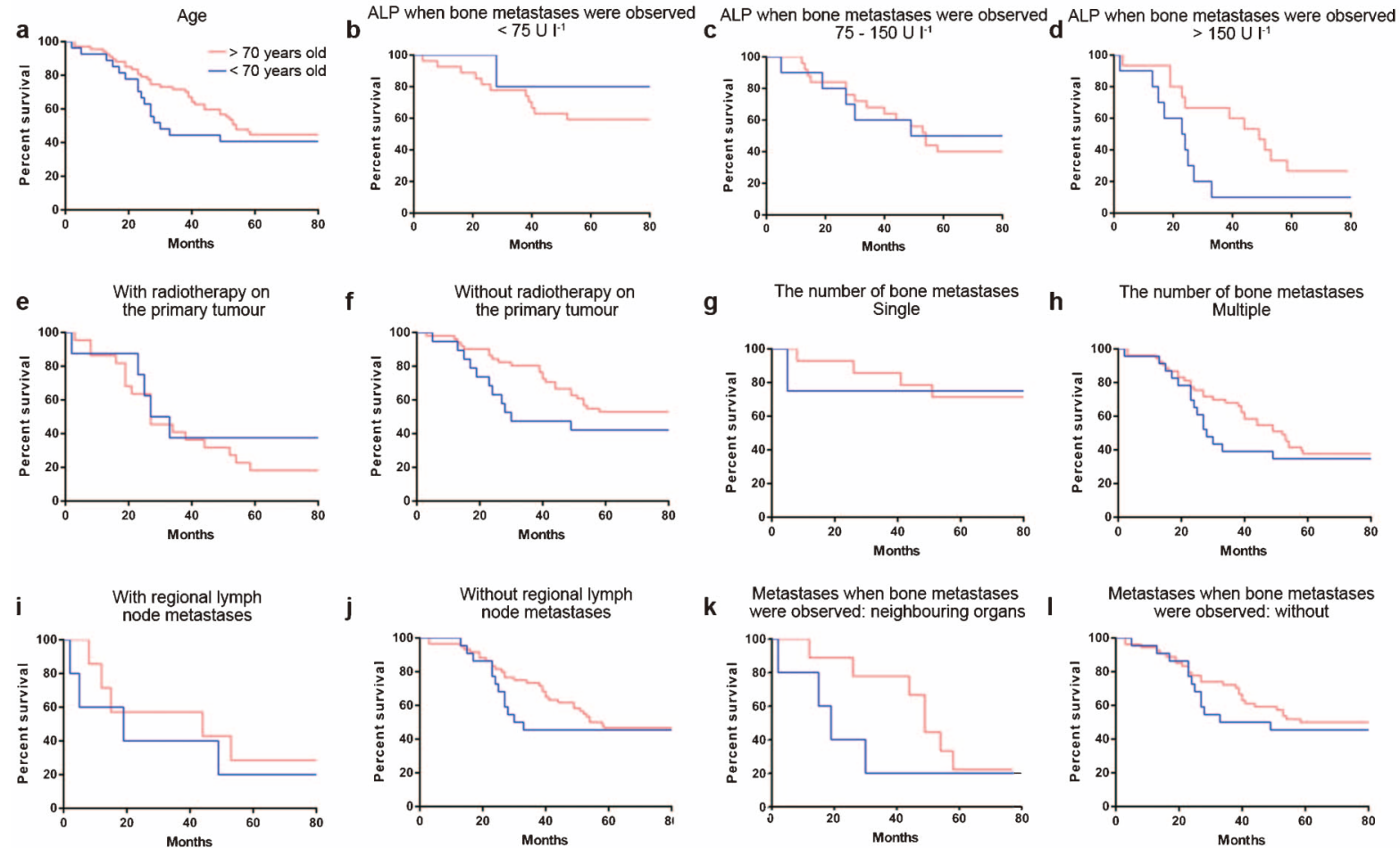

Figure 2 Comparisons of survival rates between older and younger patients. The overall survival rates between older and younger patients (a). The comparisons of survival rates between older and younger patients with different ALP levels (b-d), with or without radiotherapy of the primary tumour (e-f), with single or multiple sites of bone metastasis $(\mathbf{g}-\mathbf{h})$ and with or without regional lymph node or neighbouring organ metastasis $(\mathbf{i}-\mathbf{I}) .{ }^{*} P<0.05 ; * *<0.01$. ALP, alkaline phosphatase.

the advantage of radiotherapy, ${ }^{6}$ and the results were similar to those of another recent study. ${ }^{15}$ However, in the current study, the older patients who received radiotherapy showed a lower survival rate when compared with the younger patients. In addition, the overall survival rate of the older patients was significantly higher compared with the younger patients. In the older patients, in addition to radiotherapy, the number of bone metastases, ALP $>150 \mathrm{ng} \mathrm{ml}^{-1}$ and lymph node or organ metastasis were all associated with the difference in the survival rate between older and younger patients. These data revealed a better outcome for older patients with PCa bone metastasis.

Prostate cancer is primarily dependent on androgen, which is secreted less and more slowly with increasing age. The benefits of androgen deprivation therapy are well recognized, and a multitude of studies have documented the benefits of androgen deprivation therapy in conjunction with other therapies. ${ }^{16}$ In our study, we observed that the overall survival rate of the older patients was higher than that of the younger patients, and the decreased androgen level in the older patients may at least partially explain this difference. In addition, osteogenic bone destruction is a characteristic of PCa with bone metastasis. ${ }^{17}$ As a result and in contrast to other cancers, metastatic sites cause less pain, fewer pathologic fractures and less spinal cord compression. Currently, the first-line treatment for PCa bone metastasis is radiotherapy, ${ }^{18,19}$ which represents an active treatment strategy. However, our study demonstrates that it is not beneficial for older patients with bone metastasis to receive radiotherapy on the primary tumour site. Radiotherapy affects not only tumour cells but also normal cells. Bone metastasis is not an important cause of death for older patients; the vulnerable homeostasis might be influenced by radiotherapy, which impairs existing diseases such as cardiovascular and respiratory system diseases in older patients. Therefore, radiotherapy on the primary tumour site for older patients should be used with caution. However, it also should be noted that the number of patients who received radiotherapy on the primary tumour site was less than that of patients who did not in this study. The result needs to be confirmed in a larger sample size in the future. But in respect to bone metastasis sites, especially on the spine or other weight bearing bones, it is universally acknowledged that radiotherapy improves the quality of life by inhibiting pain and decreasing the rate of pathological fracture.

\section{CONCLUSION}

In conclusion, Gleason score, radiotherapy, the number of bone metastases, the ALP level and neighbouring or distant organ and regional lymph node metastases were associated with the survival of older patients with bone metastases from PCa. The survival rate was higher among the older patients compared with the younger patients. These data may serve as a guide for building clinical prediction models in future studies.

\section{AUTHOR CONTRIBUTIONS}

$\mathrm{CY}$ analysed, interpreted the clinical data and wrote the manuscript. $\mathrm{JH}$ designed the study, analysed the data, revised the manuscript and supervised the project. RMR designed the study and supervised the project. GSQ collected patients' clinical data. All authors vouch for the respective data and analysis, approved the final version and agreed to publish the manuscript.

\section{COMPETING FINANCIAL INTERESTS}

All authors declare that there are no competing financial interests.

1 Siegel R, Ward E, Brawley O, Jemal A. Cancer statistics, 2011: the impact of eliminating socioeconomic and racial disparities on premature cancer deaths. $C A$ Cancer J Clin 2011; 61: 212-36. 
2 Paller CJ, Carducci MA, Philips GK. Management of bone metastases in refractory prostate cancer-role of denosumab. Clin Interv Aging 2012; 7: 363-72.

3 Rigaud J, Tiguert R, Le Normand L, Karam G, Glemain P et al. Prognostic value of bone scan in patients with metastatic prostate cancer treated initially with androgen deprivation therapy. J Urol 2002; 168: 1423-6.

4 Groot MT, Boeken Kruger CG, Pelger RC, Uyl-de Groot CA. Costs of prostate cancer, metastatic to the bone, in the Netherlands. Eur Urol 2003; 43: 226-32.

5 Tangen CM, Faulkner JR, Crawford ED, Thompson IM, Hirano D et al. Ten-year survival in patients with metastatic prostate cancer. Clin Prostate Cancer 2003; 2: 41-5.

$6 \mathrm{He} \mathrm{J}$, Zeng ZC, Yang P, Chen B, Jiang W et al. Clinical features and prognostic factors for patients with bone metastases from prostate cancer. Asian J Androl 2012; 14: 505-8.

7 Imai $\mathrm{K}$, Suzuki T, Shimizu T, Yamanaka $\mathrm{H}$, Kanetake $\mathrm{H}$ et al. Analysis of survival of prostate cancer patients in Japan and the U.S.A. Adv Exp Med Biol 1992; 324: 29-38.

8 Glass TR, Tangen CM, Crawford ED, Thompson I. Metastatic carcinoma of the prostate: identifying prognostic groups using recursive partitioning. J Urol 2003; 169: 164-9.

9 Yigitbasi O, Ozturk U, Goktug HN, Gucuk A, Bakirtas H. Prognostic factors in metastatic prostate cancer. Urol Oncol 2011; 29: 162-5.

10 Chen CH, Tzai TS, Huang SP, Wu HC, Tai HC et al. Clinical outcome of Taiwanese men with metastatic prostate cancer compared with other ethnic groups. Urology 2008; 72: 1287-92.

11 Tangen CM, Hussain MH, Higano CS, Eisenberger MA, Small EJ et al. Improved overal survival trends of men with newly diagnosed M1 prostate cancer: a SWOG phase III trial experience (S8494, S8894 and S9346). J Urol 2012; 188: 1164-9.
12 Jorgensen $\mathrm{T}$, Kanagasingam $\mathrm{Y}$, Kaalhus $\mathrm{O}$, Tveter KJ, Bryne M et al. Prognostic factors in patients with metastatic (stage D2) prostate cancer: experience from the Scandinavian Prostatic Cancer Group Study-2. J Urol 1997; 158: 164-70.

13 Cheville JC, Tindall D, Boelter C, Jenkins R, Lohse CM et al. Metastatic prostate carcinoma to bone: clinical and pathologic features associated with cancer-specific survival. Cancer 2002; 95: 1028-36.

14 Saeter G, Fossa SD, Ous S, Blom GP, Kaalhus O. Carcinoma of the prostate with soft tissue or non-regional lymphatic metastases at the time of diagnosis: a review of 47 cases. Br J Urol 1984; 56: 385-90.

15 Guarneri A, Botticella A, Filippi AR, Ruggieri A, Piva C et al. Radical radiotherapy in high-risk prostate cancer patients with high or ultra-high initial PSA levels: a single institution analysis. J Cancer Res Clin Oncol 2013; 39: 1141-7.

16 Bourke L, Kirkbride P, Hooper R, Rosario AJ, Chico TJ et al. Endocrine therapy in prostate cancer: time for reappraisal of risks, benefits and cost-effectiveness? $\mathrm{Br} J$ Cancer 2013; 108: 9-13.

17 Yuen HF, Kwok WK, Chan KK, Chua CW, Chan YP et al. TWIST modulates prostate cancer cell-mediated bone cell activity and is upregulated by osteogenic induction. Carcinogenesis 2008; 29: 1509-18.

18 Second-line treatment of metastatic prostate cancer. Prednisone and radiotherapy for symptom relief. Prescrire Int 2013; 22: 74-8.

19 Chowdhury S, Kirby R. Advances in the treatment of metastatic prostate cancer. Practitioner 2013; 257: 15-8, 2 . 